\title{
Expression of neural cell adhesion molecule in salivary adenoid cystic carcinoma and its correlation with perineural invasion
}

\author{
JINGBIAO SHANG ${ }^{1,2}$, LIMING SHENG $^{1}$, KEJING WANG $^{2}$, YONGJIE SHUI $^{1}$ and QICHUN WEI $^{1}$ \\ ${ }^{1}$ Department of Radiation Oncology, The Second Affiliated Hospital, Cancer Institute, Zhejiang University \\ School of Medicine, Hangzhou 310009; ${ }^{2}$ Department of Head and Neck Surgery, \\ Zhejiang Cancer Hospital, Hangzhou 310016, P.R. China
}

Received May 29, 2007; Accepted July 25, 2007

\begin{abstract}
The expression of neural cell adhesion molecule (NCAM) was analyzed in immunohistochemical preparations from adenoid cystic carcinoma. The goal was to evaluate whether NCAM expression could be used as a biological marker for the perineural invasion of adenoid cystic carcinoma in the head and neck. The presence of perineural invasion and NCAM expression was evaluated in samples from 49 patients. Perineural invasion was identified in 33 of them $(67 \%)$. A high incidence of perineural invasion was found in adenoid cystic carcinoma in the parotid, hard palate, maxillary sinus and oral cavity. Positive NCAM staining was observed in 28 of 49 patients $(57 \%)$. Of the 28 patients with NCAM staining, perineural invasion was identified in $24(86 \%)$. In contrast, only $9(43 \%)$ of the 21 tumors without NCAM staining had perineural invasion. The difference in NCAM expression between cases with and without perineural invasion was statistically significant $(\mathrm{p}<0.01)$. When positive NCAM staining was used to estimate the presence of perineural invasion, the sensitivity was 73 and the specificity $75 \%$. Histopathologic nodal involvement was found in 6 of 18 cases in which neck dissection had been performed. All 6 cases displayed positive NCAM staining, and 5 displayed perineural invasion in the primary adenoid cystic carcinoma. In conclusion, NCAM expression can, to a certain extent, be used as a predictor of perineural invasion in adenoid cystic carcinoma. Moreover, lymph node metastases could serve as a clinical indicator for perineural invasion and for NCAM expression.
\end{abstract}

\section{Introduction}

Adenoid cystic carcinoma is a common tumor of the salivary gland which accounts for $10-15 \%$ of major salivary gland

Correspondence to: Dr Qichun Wei, Department of Radiation Oncology, the Second Affiliated Hospital, Cancer Institute, Zhejiang University School of Medicine, Hangzhou 310009, P.R. China E-mail: qichun_wei@zju.edu.cn

Key words: neural cell adhesion molecule, salivary adenoid cystic carcinoma, perineural invasion, immunohistochemistry malignancies (1-3). Incidence of this cancer is even higher among malignant minor salivary gland tumors $(3,4)$. A prominent feature of adenoid cystic carcinoma is its affinity for nerves or blood vessels. Perineural invasion has been accepted as an important risk factor for local recurrence and lymph nodes or distant metastases (5). Other studies have implicated perineural invasion with decreased survival $(6,7)$. Once the tumor invades the perineural space, it appears to migrate in the nerve sheet for a long distance and provides a nidus for recurrence and metastases after surgery. For this reason, wider margins of resection and post-operative radiotherapy have been recommended for patients with perineural invasion (7). However, clinical examination and radiographic findings are often unreliable methods for its prediction, and biopsy samples may be too small to demonstrate its presence. There is a need for biological markers expressed by adenoid cystic carcinoma that can predict a propensity for perineural invasion.

Neural cell adhesion molecule (NCAM), a membrane receptor of the immunoglobulin supergene family, is recognized to play an important role in perineural invasion in various neoplasms such as pancreatic, bile duct and gallbladder cancer, and melanomas (8-10). The literature on NCAM expression in adenoid cystic carcinoma is sparse. In the few clinical reports found in the English literature, the expression of NCAM in adenoid cystic carcinoma has been reported to vary between 25 and $100 \%$ (11-14). Meanwhile, the function of NCAM as a determinant promoting the spread of adenoid cystic carcinoma along the nerves remains to be determined.

In the present study, the expression of NCAM was investigated immunohistochemically in 49 cases of salivary adenoid cystic carcinoma. The aim was to add to the body of data on the subject and to determine the role of NCAM in perineural invasion.

\section{Materials and methods}

Patients and samples. Patients with salivary adenoid cystic carcinoma who were diagnosed and treated at Zhejiang Cancer Hospital, Hangzhou, China between 1996 and 2006 were enrolled in the study. Those who had received pre-operative radiotherapy or chemotherapy were excluded. In total, 49 patients with high quality material were finally included. All cases were treated with surgical resection, and neck lymph 
Table I. Tumor and patient characteristics $(n=49)$.

\begin{tabular}{ll}
\hline Characteristics & No. of patients $(\%)$ \\
\hline Historical pattern & \\
$\quad$ Tubular & $20(41)$ \\
Trabecular & $4 \quad(8)$ \\
Cribriform & $13(27)$ \\
$\quad$ Solid & $12(24)$ \\
T-stages & \\
T1 & $5(10)$ \\
T2 & $21(43)$ \\
T3 & $9(18)$ \\
T4 & $14(29)$ \\
Neck dissection done & $18(37)$ \\
Histopathologic nodal involvement & $6(12)$ \\
Gender & \\
Male & $22(45)$ \\
Female & $27(55)$ \\
Median age (years) & \\
Male & 50 \\
Female & 44 \\
\hline
\end{tabular}

node dissection was performed in 18 of the 49 patients. Clinical information was obtained from hospital records and included patient age and sex, the location of the primary tumor, disease stage, histological pattern and nodal involvement. Patient and tumor characteristics of the analyzed cases are shown in Table I.

NCAM-staining. NCAM was assessed by immunohistochemistry. Briefly, the tissues were fixed in $4 \%$ buffered formalin, processed and embedded in paraffin. Sections, $4 \mu \mathrm{m}$ thick, were then cut and deparaffinized in xylene and hydrated through graded concentrations of ethanol to distilled water. After the deparaffinization of the sections, endogenous peroxidase was blocked with $3 \% \mathrm{H}_{2} \mathrm{O}_{2}$ in absolute methanol. For antigen retrieval, sections were submitted to high temperatures and pressure for $5 \mathrm{~min}$ using the Tris-EDTA buffer (pH 9.0). The sections were then blocked with normal goat serum. The primary mouse monoclonal antibody directed against NCAM (clone 123C3, Zymed labs, South San Francisco, CA, USA) was diluted 1:100 and incubated overnight at $4^{\circ} \mathrm{C}$. As a secondary antibody, Envision+ (DAKO, Glostrup, Denmark), a dextran polymers conjugating a large number of goat antibodies against mouse immunoglobulins and horseradish peroxidase, was used to increase the sensitivity of immunodetection (15). All slides were developed in $0.05 \%$ diamino benzidine (Sigma, St. Louis, MO, USA) for $5 \mathrm{~min}$ and counterstained in Harris haematoxylin (Sigma). Finally, the slides were dehydrated through graded alcohol to xylene and mounted in organic mounting medium. Tissue sections of natural killer/T-cell lymphoma (known to
Table II. Presence of perineural invasion by site of adenoid cystic carcinoma.

\begin{tabular}{lcc}
\hline Primary site & $\begin{array}{c}\text { No. of } \\
\text { tumors }\end{array}$ & $\begin{array}{c}\text { No. of perineural } \\
\text { invasion }(\%)\end{array}$ \\
\hline $\begin{array}{l}\text { Major salivary gland } \\
\text { Parotid }\end{array}$ & 11 & $8(72)$ \\
$\quad$ Submaxillary & 6 & $3(50)$ \\
$\quad$ Sublingual & 3 & $2(67)$ \\
Minor salivary gland & & \\
$\quad$ Oral cavity & 7 & $5(71)$ \\
$\quad$ Soft palate & 2 & $1(50)$ \\
Hard palate & 7 & $6(86)$ \\
Nasal cavity & 3 & $1(33)$ \\
Maxillary sinus & 6 & $2(83)$ \\
$\quad$ Nasopharynx & 4 & \\
\hline
\end{tabular}

be positive for NCAM staining) served as positive controls. Nerve tissue included in the specimens served as an internal positive control. As a negative control, the first antibody was replaced with PBS during staining. These controls were run in parallel. NCAM staining was evaluated using a scale of $0-3+$, with 0 corresponding to completely negative tumor cells and $3+$ corresponding to the strongest staining of tumor cells (16).

Assessment of perineural invasion. The presence of perineural invasion was assessed in all specimens by 2 independent observers blind to patient status. Any differences were resolved by joint review and consultation with a third observer. For each tumor sample, 5 haematoxylin and eosin stained tissue sections from different tumor locations were checked. Specimens demonstrating encasement of the nerve or violation of the nerve sheath were recorded as positive.

Statistical Analysis. The $\chi^{2}$ statistical analysis test was used to detect the correlation between NCAM staining, perineural invasion and historical pattern.

\section{Results}

Perineural invasion in adenoid cystic carcinoma. Perineural invasion was identified in 33 of 49 patients $(67 \%)$. The presence of perineural invasion and its relationship between the primary sites is shown in Table II. High incidence of perineural invasion was found in adenoid cystic carcinoma in the parotid, hard palate, maxillary sinus and oral cavity.

NCAM expression and its relation to perineural invasion. Intensive expression ( 2 or $3+$ ) of NCAM was found in 5 of the adenoid cystic carcinoma analyzed; only 1 of these cases showed $3+$ in the tumor. Of the cases, 23 had 1+ NCAM staining. In total, positive NCAM staining was observed in 28 of the 49 patients $(57 \%)$. An example of NCAM expression 


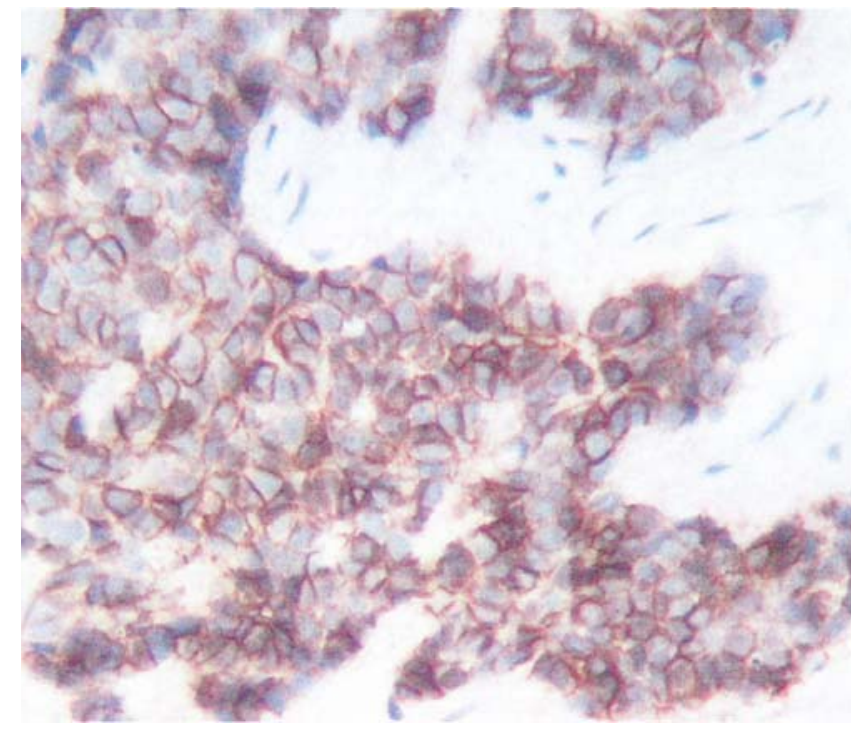

Figure 1. Example of immunohistochemical brown stainings of neural cell adhesion molecule in salivary adenoid cystic carcinoma. Weak blue nuclear stainings with haematoxylin (original magnification $\mathrm{x} 200$ ).

Table III. Expression of NCAM in relation to presence of perineural invasion in adenoid cystic carcinoma.

\begin{tabular}{lcc}
\hline $\begin{array}{l}\text { NCAM } \\
\text { staining }\end{array}$ & $\begin{array}{c}\text { No. with } \\
\text { perineural invasion }\end{array}$ & $\begin{array}{c}\text { No. without } \\
\text { perineural invasion }\end{array}$ \\
\hline Positive & 24 & 4 \\
Negative & 9 & 12 \\
\hline
\end{tabular}

$\chi^{2}=10.02 ; \mathrm{p}<0.01$.

(scored as 2+) is documented in Fig. 1. Of the 28 patients with NCAM staining, perineural invasion was identified in 24 $(86 \%)$. In contrast, only $9(43 \%)$ of the 21 tumors without NCAM staining showed perineural invasion. A statistically significant association between NCAM expression and perineural invasion is shown in Table III. When used to estimate the presence of perineural invasion, the sensitivity of positive NCAM staining was 73 and the specificity $75 \%$.

Of the 5 patients with intensive NCAM staining, perineural invasion was identified in $4(80 \%)$. The corresponding percentage of patients with 1+ NCAM staining was $87 \%$ (20/23). No difference was observed among the positive NCAM staining cases.

NCAM expression and cancer staging. The relationship between the expression of NCAM and T stages (primary tumor) is shown in Table IV. Similar NCAM staining was found regardless of $\mathrm{T}$ stage. Neck dissection was performed in 18 of the 49 patients, with histopathologic nodal involvement found in 6 of the 18 cases. Positive NCAM staining was present in all 6 cases, while none of the patients with negative NCAM staining had lymph node metastases. Perineural invasion was present in $11(61 \%)$ of the 18 cases
Table IV. Correlation between NCAM expression and T stages in adenoid cystic carcinoma.

\begin{tabular}{lcc}
\hline T stage & $\begin{array}{c}\text { No. of } \\
\text { tumors }\end{array}$ & $\begin{array}{c}\text { No. of } \\
\text { positive NCAM (\%) }\end{array}$ \\
\hline T1 & 5 & $3(60)$ \\
T2 & 21 & $12(57)$ \\
T3 & 9 & $5(56)$ \\
T4 & 14 & $8(57)$ \\
\hline
\end{tabular}

Table V. Correlation between NCAM expression and histological pattern of adenoid cystic carcinoma.

\begin{tabular}{lcc}
\hline $\begin{array}{l}\text { Histological } \\
\text { pattern }\end{array}$ & $\begin{array}{c}\text { No. of } \\
\text { tumors }\end{array}$ & $\begin{array}{c}\text { No. of } \\
\text { positive NCAM (\%) }\end{array}$ \\
\hline Cribriform & 13 & $7(54)$ \\
Tubular-Trabecular & 24 & $17(71)$ \\
Solid & 12 & $4(33)$ \\
\hline$\chi^{2}=4.67 ; \mathrm{p}>0.05$. & & \\
\hline
\end{tabular}

with neck dissection. Five of the $11(45 \%)$ patients with perineural invasion showed lymph node metastases, while only 1 of 7 patients without perineural invasion had lymph node metastases.

NCAM expression and histological patterns. The expression of NCAM was seen in $71 \%(17 / 24)$ of cases with tubulartrabecular pattern, whereas NCAM expression tended to be low (4/12) in solid pattern adenoid cystic carcinoma. However, there was no statistically significant association between the histological pattern and expression of NCAM (Table V).

\section{Discussion}

This study was done with the aim of determining whether NCAM expression can be used as a biological marker of perineural invasion in adenoid cystic carcinoma of the head and neck, in order to estimate the presence of perineural invasion before surgical intervention. The information could also be helpful in deciding whether to recommend postoperative radiation therapy.

NCAM, a member of the immunoglobulin supergene family, is a glycoprotein that mediates homophilic binding between neighboring cells and heterophilic interactions between cells and extracellular matrix components $(17,18)$. NCAM is considered to be an important signal transduction receptor molecule, able to modulate diverse biological 
properties including proliferation, adhesion and migration $(19,20)$.

It has been reported that NCAM plays an important role in perineural invasion in various neoplasms, such as pancreatic, bile duct and gallbladder cancer, and melanomas (8-10). In a report on 76 patients with squamous cell carcinoma of the head and neck (SCCHN) (21), NCAM expression was demonstrated in half the cases and the incidence of NCAM expression was significantly associated with perineural invasion. In another report on SCCHN, Vural et al (16) found that $93 \%$ of specimens with perineural invasion showed evidence of NCAM expression, while only $36 \%$ of specimens without perineural invasion expressed NCAM. Literature on NCAM expression in adenoid cystic carcinoma is rare. In clinical reports which can be found through Medline, the expression of NCAM in adenoid cystic carcinoma has been reported to be $89,100,31$ and 25\% (11-14). Gandour-Edwards et al investigated 18 cases in which adenoid cystic carcinoma invaded the skull base. Perineural invasion was identified in $15(83 \%)$ of patients, and NCAM expression in $16(89 \%)$ of tumors. In cases with perineural invasion, NCAM expression was found in $93 \%$ of specimens. It has been postulated that the role of NCAM is to act as a neurodeterminant that facilitates the spread of adenoid cystic carcinoma along the nerves. Hutcheson et al reported uniform NCAM positive staining in adenoid cystic carcinoma regardless of perineural invasion state. Recently, relatively low NCAM expressions of 31 and $25 \%$ were reported in other studies, with no difference being found between patients with and without perineural invasion. The function of NCAM as a determinant promoting perineural invasion in adenoid cystic carcinoma remains unclear. In this study, we reported on a series of 49 patients with adenoid cystic carcinoma. To date, it is the largest series on adenoid cystic carcinoma with NCAM expression as the focus. NCAM expression was found in $57 \%$ of cases, as well as a significant correlation between NCAM expression and perineural invasion in adenoid cystic carcinoma. With reasonable sensitivity $(73 \%)$ and specificity $(75 \%)$, NCAM expression can, to a certain extent, be used as a predictor of perineural invasion in adenoid cystic carcinoma.

In the present study, tumors with different $\mathrm{T}$ stages showed similar NCAM staining. Primary tumor status had no impact on the expression of NCAM. However, in the cases with lymph node metastases, positive NCAM staining was detected in all primary lesions and perineural invasion identified in most cases, highlighting the relationship between lymph node metastases and NCAM expression/perineural invasion. Even though, in this study, only a small number of patients had positive lymph nodes - meaning the statistical sample may have been too low for conclusive results - lymph node metastases could also potentially serve as a clinical indicator for perineural invasion and NCAM expression.

\section{Acknowledgments}

The authors thank Gu Zhang and Jinlin Hu for help with the immunohistochemical stainings and associate professor Yi Dai at Zhejiang Medical College for statistical analysis. The authors acknowledge economic support by a grant from the National Natural Science Foundation of China (Q. Wei, No. 30470501 .

\section{References}

1. Spiro RH: Salivary neoplasms: overview of a 35-year experience with 2,807 patients. Head Neck Surg 8: 177-184, 1986.

2. Ostman J, Anneroth G, Gustafsson H and Tavelin B: Malignant salivary gland tumours in Sweden 1960-1989, an epidemiological study. Oral Oncol 33: 169-176, 1997.

3. Lima SS, Soares AF, Amorim RF and Freitas RD: Epidemiologic profile of salivary gland neoplasms: analysis of 245 cases. Rev Bras Otorrinolaringol (Engl Ed) 71: 335-340, 2005.

4. Neely MM, Rohrer MD and Young SK: Tumors of minor salivary glands and the analysis of 106 cases. J Okla Dent Assoc 86: 50-52, 1996.

5. Vrielinck LJ, Ostyn F, van Damme B, van den Bogaert W and Fossion E: The significance of perineural spread in adenoid cystic carcinoma of the major and minor salivary glands. Int J Oral Maxillofac Surg 17: 190-193, 1988.

6. Issing PR, Hemmanouil I, Wilkens L, Karstens H and Lenarz T: Long term results in adenoidcystic carcinoma. Laryngorhinootologie 81: 98-105, 2002.

7. Garden AS, Weber RS, Morrison WH, Ang KK and Peters LJ: The influence of positive margins and nerve invasion in adenoid cystic carcinoma of the head and neck treated with surgery and radiation. Int J Radiat Oncol Biol Phys 32: 619-626, 1995.

8. Kameda K, Shimada H, Ishikawa T, Takimoto A, Momiyama N, Hasegawa S, Misuta K, Nakano A, Nagashima Y and Ichikawa Y: Expression of highly polysialylated neural cell adhesion molecule in pancreatic cancer neural invasive lesion. Cancer Lett 137: 201-207, 1999

9. Seki H, Koyama K, Tanaka J, Sato Y and Umezawa A: Neural cell adhesion molecule and perineural invasion in gallbladder cancer. J Surg Oncol 58: 97-100, 1995.

10. Seki H, Tanaka J, Sato Y, Kato Y, Umezawa A and Koyama K: Neural cell adhesion molecule (NCAM) and perineural invasion in bile duct cancer. J Surg Oncol 53: 78-83, 1993.

11. Gandour-Edwards R, Kapadia SB, Barnes L, Donald PJ and Janecka IP: Neural cell adhesion molecule in adenoid cystic carcinoma invading the skull base. Otolaryngol Head Neck Surg 117: 453-458, 1997.

12. Hutcheson JA, Vural E, Korourian S and Hanna E: Neural cell adhesion molecule expression in adenoid cystic carcinoma of the head and neck. Laryngoscope 110: 946-948, 2000.

13. Perschbacher K, Jackson-Boeters L and Daley T: The adhesion molecules NCAM, HCAM, PECAM-1 and ICAM-1 in normal salivary gland tissues and salivary gland malignancies. J Oral Pathol Med 33: 230-236, 2004.

14. Xue F, Zhang Y, Liu F, Jing J and Ma M: Expression of IgSF in salivary adenoid cystic carcinoma and its relationship with invasion and metastasis. J Oral Pathol Med 34: 295-297, 2005.

15. Sabattini E, Bisgaard K, Ascani S, Poggi S, Piccioli M, Ceccarelli C, Pieri F, Fraternali-Orcioni G and Pileri SA: The EnVision++ system: a new immunohistochemical method for diagnostics and research. Critical comparison with the APAAP, ChemMate, CSA, LABC, and SABC techniques. J Clin Pathol 51: 506-511, 1998.

16. Vural E, Hutcheson J, Korourian S, Kechelava S and Hanna E: Correlation of neural cell adhesion molecules with perineural spread of squamous cell carcinoma of the head and neck. Otolaryngol Head Neck Surg 122: 717-720, 2000.

17. Walsh FS and Doherty P: Neural cell adhesion molecules of the immunoglobulin superfamily: role in axon growth and guidance. Annu Rev Cell Dev Biol 13: 425-456, 1997.

18. Burg MA, Halfter W and Cole GJ: Analysis of proteoglycan expression in developing chicken brain: characterization of a heparan sulfate proteoglycan that interacts with the neural cell adhesion molecule. J Neurosci Res 41: 49-64, 1995.

19. Prag S, Lepekhin EA, Kolkova K, Hartmann-Petersen R, Kawa A, Walmod PS, Belman V, Gallagher HC, Berezin V, Bock E and Pedersen N: NCAM regulates cell motility. J Cell Sci 115: 283-292, 2002.

20. Panicker AK, Buhusi M, Thelen K and Maness PF: Cellular signalling mechanisms of neural cell adhesion molecules. Front Biosci 8:D900-D911, 2003.

21. McLaughlin RB Jr, Montone KT, Wall SJ, Chalian AA, Weinstein GS, Roberts SA, Wolf PF and Weber RS: Nerve cell adhesion molecule expression in squamous cell carcinoma of the head and neck: a predictor of propensity toward perineural spread. Laryngoscope 109: 821-826, 1999. 